\title{
Virologic versus immunologic monitoring and the rate of accumulated genotypic resistance to first-line antiretroviral drugs in Uganda
}

Steven J Reynolds ${ }^{1,2,8^{*}}$, Hakim Sendagire ${ }^{3,4}$, Kevin Newell ${ }^{5}$, Barbara Castelnuovo ${ }^{6}$, Immaculate Nankya ${ }^{7}$, Moses Kamya ${ }^{6}$, Thomas C Quinn ${ }^{1,2}$, Yukari C Manabe ${ }^{2,6}$ and Andrew Kambugu ${ }^{6}$

\begin{abstract}
Background: Viral load monitoring (VLM) to identify individuals failing antiretroviral therapy (ART) is not widely available in resource-limited settings. We compared the genotypic resistance patterns between clients with VLM versus immunological monitoring (IM).

Methods: Between 2004-2008, 559 ART naïve clients were enrolled in a prospective cohort, initiated on ART, and monitored with viral load (VL) and CD4+ cell counts every 6 months (VLM group). From February 2008 through June 2009, 998 clients on ART for 36-40 months (corresponding to the follow-up time of the VLM group) at the same clinic and monitored with CD4+ cell counts every 6 months were recruited into a cross sectional study (IM group). Samples from VLM clients at 12, 24 and 36 months and IM clients at 36-40 months with VL > 2000 copies/ $\mathrm{ml}$ underwent genotypic drug resistance testing.

Results: Baseline characteristics were similar. Virologic failure (VL $>400$ copies/ml) at 12, 24 and 36 months in the VLM group were 12\%, 6\% and 8\% respectively, and in the IM group 10\% at 36-40 months. Samples from 39 VLM and 70 IM clients were genotyped. 23/39 (59\%) clients in the VLM group (at 12, 24 or 36 months) compared to 63/ 70 (90\%) in the IM group, $(P<0.0001)$ had at least 1 non-nucleoside reverse transcriptase mutation. 19/39 (49\%) of VLM clients had an M184V mutation compared to 61/70 (87\%) in the IM group $(P<0.0001)$. Only 2/39 (5\%) of VLM clients developed thymidine analogue mutations compared to $34 / 70(49 \%)$ of IM clients $(P<0.0001)$.
\end{abstract}

Conclusions: Routine VL monitoring reduced the rate of accumulated genotypic resistance to commonly used ART in Uganda.

Keywords: HIV-1, Antiretroviral therapy, Drug resistance

\section{Background}

Antiretroviral treatment (ART) programs have scaled up to reach over 5.2 million HIV-infected individuals in need of life-saving treatment in low and middle income countries by the end of 2009 [1]. Most of these individuals live in settings where laboratory monitoring is limited and treatment failure is determined using either clinical or immunological criteria. Concern has been raised regarding the performance of clinical and

\footnotetext{
*Correspondence: sjreynolds@niaid.nih.gov

'Division of Intramural Research, National Institute of Allergy and Infectious Diseases, National Institutes of Health, Bethesda, MD, USA

2Johns Hopkins University School of Medicine, Baltimore, MD, USA

Full list of author information is available at the end of the article
}

immunologic treatment failure criteria to correctly identify individuals with virologic failure [2-7]. Prolonged virologic failure may result in accumulation of resistance mutations to commonly used first-line ART as observed in Malawi and elsewhere [8,9]. This could compromise second-line treatment outcomes and also increase the risk of transmitted HIV drug resistance.

Failure to achieve or maintain virologic suppression for prolonged periods of time may lead to the sequential development of thymidine analogue mutations (TAMs) in clients taking nucleoside reverse transcriptase inhibitors (NRTIs) such as zidovudine (ZDV) or stavudine (d4T) as part of their ART regimens [9-12]. The accumulation of 3 or 4 TAMs confers drug resistance across

\section{Ciomed Central}


the NRTI class of antiretroviral (ARV) drugs and has implications for future ART regimens, particularly in resource-limited settings (RLS) where second-line ART options are limited. ZDV, tenofovir (TDF) and to a lesser extent $\mathrm{d} 4 \mathrm{~T}$ are currently used as the nucleoside backbone of the first-line and some second-line regimens in sub-Saharan Africa and other RLS areas.

The objective of our study was to compare genotypic resistance patterns (with particular attention to TAMs) among a group of clients on first-line ART at an urban clinic in Kampala, Uganda, who were monitored both clinically, immunologically and virologically as part of a research cohort study to a similar group of clients attending the same clinic who were monitored only clinically and immunologically.

\section{Methods}

\section{Study setting}

The Infectious Diseases Institute (IDI) of Makerere University College of Health Sciences (Kampala, Uganda) is a center of excellence in the delivery of HIV clinical care, research and training, with over 10,000 active clients registered including over 6,500 receiving ART. Eligibility criteria for ART at the time of this study (February 2008 through June 2009) were $\mathrm{CD} 4<=200$ cells/ul or WHO stage IV disease; first-line regimens included stavudine or zidovudine plus lamivudine and nevirapine or efavirenz.

\section{Study population}

\section{The IDI routine care clinic (immunological monitoring (IM)}

group)

Clients attending the routine care clinic who become eligible for ART are offered adherence counseling prior to ART and also during follow-up if deemed necessary by clinicians. Clients are seen monthly for follow-up assessments, drug refills and monitored through prospective CD4+ cell counts every 6 months. Treatment failure was assessed according to the WHO immunologic or clinical criteria for treatment failure [13]. A more detailed description of this population is presented elsewhere [14].

\section{The IDI research cohort (viral load monitoring (VLM) group)}

A nested research cohort within the routine care clinic was established in 2004, with a consecutive prospective enrollment of 559 ART-naïve clients who initiated ART from April 2004 through April 2005; clients enrolled in this cohort are monitored through both CD4+ counts and viral load measurement (Amplicor HIV-1 Monitor PCR Test, version 1.5; Roche Diagnostic, GmbH Molecular Systems, Pleasanton, CA with a detection limit of 400 copies $/ \mathrm{ml}$ ), every 6 months; plasma is also stored for future investigations. A more detailed description of the study procedures and data collection has been presented elsewhere [15]. In brief, ART-eligible adults ( $>=18$ years of age) were enrolled in the study if they fulfilled the following eligibility criteria: 1) confirmed HIV type 1 infection; 2) regular attendance at clinic visit, based on at least 2 clinic visits within the previous 6 months; 3 ) stable residence within a $20-\mathrm{km}$ radius of Kampala; 4) willingness to be followed at the Infectious Diseases Institute for at least 2 years; and 5) eligibility for ART according to the World Health Organization (WHO) 2003 and Uganda Ministry of Health (ie, a CD4+ cell count $<=200$ cells/ul or WHO stage IV), and 6) provision of written informed consent. Clients were seen every three months by the study clinicians and monthly for routine clinical visits and drug refills. Clients were considered for switch to second line ART if they had two HIV VL $>1000$ copies $/ \mathrm{ml}$ on two consecutive measurements.

\section{Study design}

From February 2008 through June 2009, a cross sectional study was conducted in the IDI routine care clinic population recruiting a convenience sample of clients who were on first-line ART regimens for 36-40 months (corresponding to the length of follow-up time reached by IDI research cohort clients). Clients still on first-line ART were consecutively recruited during their monthly follow-up visits. After providing written informed consent, blood was drawn for HIV viral load (VL), CD4+ cell count and plasma was stored for genotyping. Genotyping was done on all clients found to have an HIV $\mathrm{VL}>2,000$ copies $/ \mathrm{ml}$ (corresponding to the sensitivity threshold of the assay). Results of the viral load and genotyping test were made available to the physicians for clinic management of their clients.

In the research cohort clients, those alive and in care at 12 months were considered eligible for this analysis. We included any research cohort client having a HIV VL of $>2000$ copies $/ \mathrm{ml}$ while still on first-line at months 12 , 24 or 36 of follow-up; however, clients who had a detectable VL above 2,000 copies/ml up to month 12 but subsequently (months 24 or beyond) became virologically suppressed without regimen change were not included in the genotyping analysis as these were considered to have early adherence problems rather than drug resistance [16]. All clients in both groups were followed-up regardless of treatment change. Genotyping was done retrospectively on stored plasma samples. Genotypic mutation patterns of the first occurrence of a viral load $>2,000$ copies $/ \mathrm{ml}$ in the research cohort clients and the mutation patterns observed among in the routine clinic population at 36-40 months were compared. All genotypes reported on VLM clients were obtained prior to switch to second-line treatment. 


\section{Statistical methods}

Baseline characteristics for both monitoring groups were compared using the Fisher exact test for categorical variables (gender, WHO staging, ART regimen) and Mann-Whitney $U$ test for continuous variables (age, CD4+ count, hemoglobin, body mass index). We used the Mann-Whitney $U$ test to compare median CD4+ count increase at follow-up. We used the t-test for proportions to assess differences in mutation rates across monitoring groups. All $P$ values are two-sided. All analyses were performed using SAS version 9.2 (SAS Institute, Cary, NC) or Stata version 11.1 (StataCorp, College Station, TX).

\section{Laboratory procedures}

Plasma was separated within 2 hours from blood draw and stored immediately at $-80^{\circ} \mathrm{C}$. HIV-1 RNA was tested with the Roche Amplicor MONITOR v1.5 assay (Roche Molecular Systems, Nutley, New Jersey, USA). HIV-1 RNA was extracted from plasma samples using a Qiagen RNA extraction method (Qiagen Inc., Chatsworth, California, USA). Polymerase gene-specific primers were used for reverse transcriptase, followed by 750 base pair pol gene encompassing amino acids 1-242 of reverse transcriptase. The PCR products was then purified using the QIAquick PCR purification kit (Qiagen, Valencia, California, USA) and sequenced using Beckman Coulter sequencing kit (Beckman Coulter Inc., Fullerton, CA). The obtained sequences were then edited using BioEdit sequence editor version 7.0.4. The cleaned sequences were then uploaded into the Stanford University HIV Drug resistance database to obtain the drug resistance profile. Genotyping assays were performed at the Joint Clinical Research Center laboratory in Kampala. For genotype analysis, mutations were generally categorized according to the International AIDS Society-USA recommendations [17].

\section{Ethics}

All study participants from the IDI routine care clinic provided written informed consent, while the participants from the IDI research cohort had already provided consent at study enrollment for future use of stored plasma. The study was approved by the National AIDS Research Council Ethics Board, the Uganda National Council for Science and Technology, and the National Institute of Allergy and Infectious Diseases Intramural Institutional Review Board.

Genbank accession numbers: KC008609 to KC008711.

\section{Results}

Four hundred forty one of the 559 research cohort clients (VLM group) were alive and in care at 12 months and included in this analysis. Nine hundred ninety-eight clients on ART for 36-40 months were recruited from the routine clinic population (IM group). Table 1 displays the demographic characteristics for the VLM and IM clients. Characteristics of patients in the VLM and IM group at ART initiation were similar; particularly median baseline CD4+ cell count was 102 (IQR 31-170) cells/ $\mu \mathrm{l}$ for VLM clients and 86 (29-154) for IM clients. The median CD4+ change from baseline to 36 months was 227 (146-336) for VLM clients and 251 (148-377) for IM clients; virologic failure rates (VL > 400 copies/ $\mathrm{ml}$ ) at 12,24 and 36 months were $12 \%, 6 \%$ and $8 \%$ respectively for VLM clients and $10 \%$ at 36-40 months for IM clients.

Samples from 39 VLM and 70 IM clients were successfully amplified and genotyped (Figure 1a \& 1b). Some VLM clients had more than one time point sent for genotyping resulting in a total of 45 unique genotypes for this group; among clients with $>1$ genotype, the first available result was used to compare VLM and IM clients as this was considered to be the time point when a switch of therapy would be considered. Among VLM clients, rates of resistance were relatively stable over 36 months follow-up (Table 2). The most common class of resistance was to the non-nucleoside reverse transcriptase inhibitors (NNRTIs) ranging from 50\% $(8 / 16)$ at 12 months, $69 \%(9 / 13)$ at 24 months and $60 \%$ $(6 / 10)$ at 36 months. Resistance to lamivudine was present among $50 \%(8 / 16)$ at 12 months, $62 \%(8 / 13)$ at 24 months and $40 \%(4 / 10)$ at 36 months. Very few VLM clients developed any resistance to the nucleoside reverse transcriptase inhibitors (NRTIs) apart from the M184V mutation.

Comparing the 39 first genotypes from VLM clients to the 70 IM clients, 23/39 (59\%) of VLM clients as opposed to $63 / 70(90 \%)$ in the IM group had at least 1 NNRTI mutation $(\mathrm{p}<0.0001)$ (Table 3$)$. Nineteen out of thirty-nine (49\%) of VLM clients had an M184V mutation compared to $61 / 70(87 \%)$ of IM clients $(\mathrm{P}<0.0001)$. Only $2 / 39(5 \%)$ of the VLM clients developed TAMS whereas $34 / 70(49 \%)$ of IM clients developed TAMS $(\mathrm{p}<0.001)$ with $7 / 70(10 \%)$ developing $>=3$ TAMS (Figure 2).

We also compared the 10 VLM clients with genotype results at 36 months to the 70 IM clients at 36 months, $6 / 10(60 \%)$ of VLM clients as opposed to 63/70 (90\%) in the IM group had at least 1 NNRTI mutation ( $p=0.1034)$. Four out of ten $(40 \%)$ of VLM clients had an M184V mutation compared to $61 / 70$ (87\%) of IM clients $(\mathrm{P}=0.0185)$. None of the VLM clients developed TAMS whereas $34 / 70$ (49\%) of IM clients developed TAMS ( $\mathrm{p}<0.0001)$.

A total of 26 clients in the VLM cohort were switched from first to second-line ART in the first 36 months of follow-up, while all patients in the IM group reached 
Table 1 Baseline Characteristics

\begin{tabular}{|c|c|c|c|}
\hline Characteristic & IM $(n=998)$ & $\operatorname{VLM}(n=441)$ & P-value \\
\hline Age (IQR yrs) & $36.2(31.5-41.3)$ & $35(30-41)$ & 0.002 \\
\hline Gender & & & 0.667 \\
\hline Female & $680(68 \%)$ & $306(69 \%)$ & \\
\hline Male & $318(32 \%)$ & $135(31 \%)$ & \\
\hline Baseline CD4 (IQR cells/ $\mu \mathrm{l})$ & $86(29-154)$ & $101.5(30.5-170)$ & 0.330 \\
\hline WHO Stage & $(n=996)$ & & $<0.0001$ \\
\hline WHO Stage I & $18(2 \%)$ & $2(1 \%)$ & \\
\hline WHO Stage II & $233(23 \%)$ & 49 (11\%) & \\
\hline WHO Stage III & $478(48 \%)$ & $257(58 \%)$ & \\
\hline WHO Stage IV & $267(27 \%)$ & $133(30 \%)$ & \\
\hline $\mathrm{Hb}(\mathrm{IQR})$ & $11.3(10.0-12.7)$ & $11.7(10.6-13.0)$ & 0.001 \\
\hline $\mathrm{BMI}\left(\mathrm{IQR} \mathrm{kg} / \mathrm{m}^{2}\right)$ & $20.2(18.2-22.5)$ & $20.2(18.3-22.6)$ & 0.917 \\
\hline ART Regimen & & & 0.726 \\
\hline $\mathrm{d} 4 \mathrm{~T} / 3 \mathrm{TC} / \mathrm{NVP}$ & $721(72 \%)$ & $325(74 \%)$ & \\
\hline $\mathrm{d} 4 \mathrm{~T} / 3 \mathrm{TC} / \mathrm{EFV}$ & $3(0.5 \%)$ & 0 & \\
\hline ZDV/3TC/NVP & $2(0.5 \%)$ & 0 & \\
\hline ZDV/3TC/EFV & $272(27 \%)$ & $116(26 \%)$ & \\
\hline
\end{tabular}

IM: Immunologically monitored; VLM: Virologically monitored; IQR: Interquartile Range; Hb: Hemoglobin; BMI: Body mass index; ART: antiretroviral therapy; d4T: stavudine; 3TC: lamivudine; ZDV: zidovudine; NVP: nevirapine; EFV: efavirenz.

36 months on first-line ART without clinicians suspecting treatment failure. The median number of VL measurements before switch was 4 and median VL prior to switch was 21,773 (IQR 5,104-210,918).

\section{Discussion}

Although the proportion of clients failing ART at 36 months was comparable between those monitored with VL and compared to those monitored with CD4+ cell counts only, the proportion of the clients with multiple resistance mutations especially TAMS was significantly higher in the IM clients. This is the first observational study directly comparing genotypic resistance profiles to commonly used antiretroviral drugs between individuals monitored with virologic compared to immunologic monitoring alone. Several studies have documented the lack of sensitivity of immunologic and clinical criteria to identify individuals failing ART raising concern regarding the potential for increased genotypic resistance to ART, which could result [3-6]. High rates of genotypic resistance to first-line ART have been observed in Malawi, Uganda and India where clinical and immunologic criteria have been used to identify treatment failure $[8,11,18]$. In addition, poor outcomes following first-line regimen failure have been documented in Malawi possibly due to advanced immunosuppression and ART resistance [19]. The results of our study add more evidence to the growing body of literature supporting some degree of viral load monitoring in RLS.
The participants enrolled in the IM cohort in this study were clients who had not been identified as having any evidence of treatment failure by clinicians. In contrast to most studies looking at resistance among clients in RLS, this was not a selected group of individuals failing by immunologic or clinical criteria and would have been left on first-line ART if VL testing was not done as part of this study. The significant degree of resistance observed in this group raises concern about the need for periodic VL monitoring in settings where none is currently available in routine care. A moderate proportion of clients had multiple TAMS possibly resulting in compromised efficacy of commonly used second-line ART regimens available in RLS.

Our study has limitations; it is not a randomized clinical trial and therefore unmeasured bias between the VLM and IM groups remains a possibility. Because the IM group was cross-sectional, we have no data on lost to follow-up or number of deaths before study enrollment; however, we would expect clients lost to care to do as poorly or worse with respect to rates of virologic failure. Clients enrolled in the VLM group followed certain inclusion criteria including living within $20 \mathrm{~km}$ of the clinic and willingness to attend to visit schedules which could also select for a more adherent population compared to the IM group. The use of stored specimens resulted in some amplification failures among the VLM group, which could possibly affect our overall results. Finally, we have no information on duration of virologic failure among the IM group to allow us to control for 


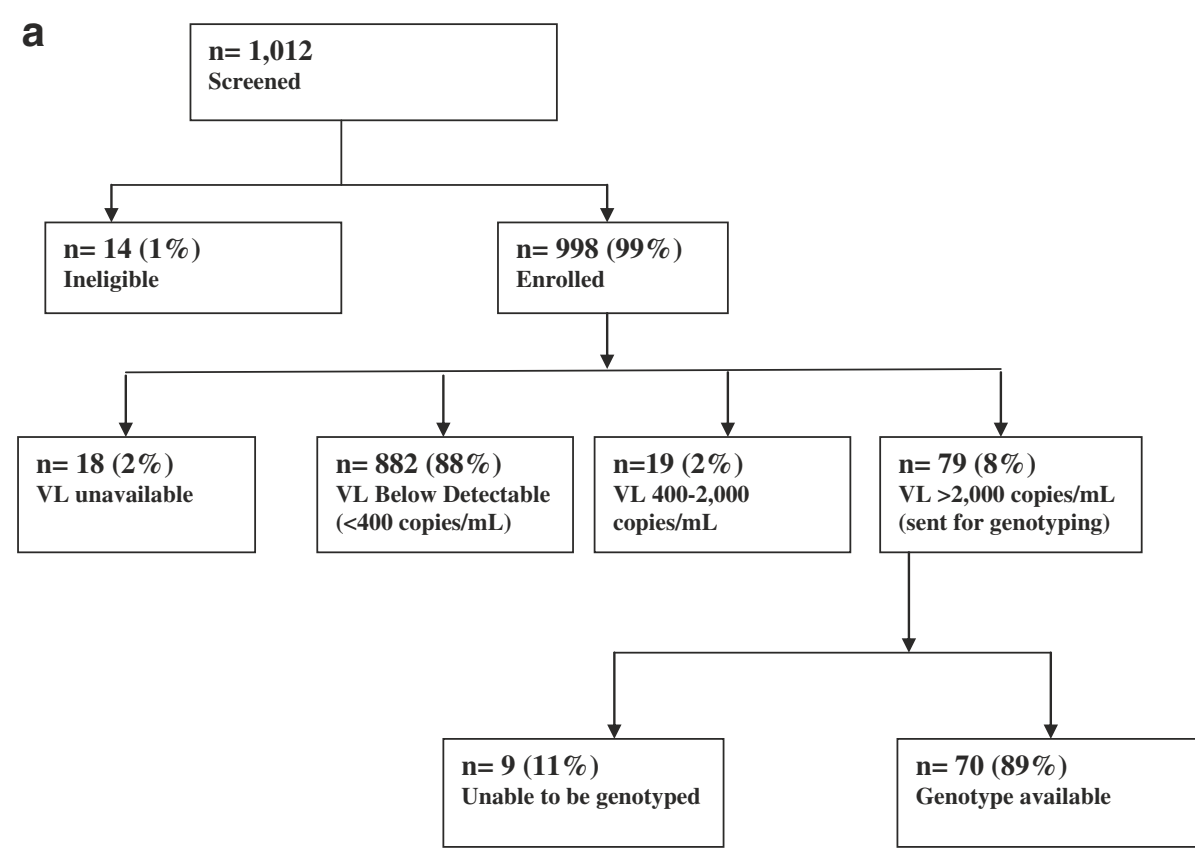

b

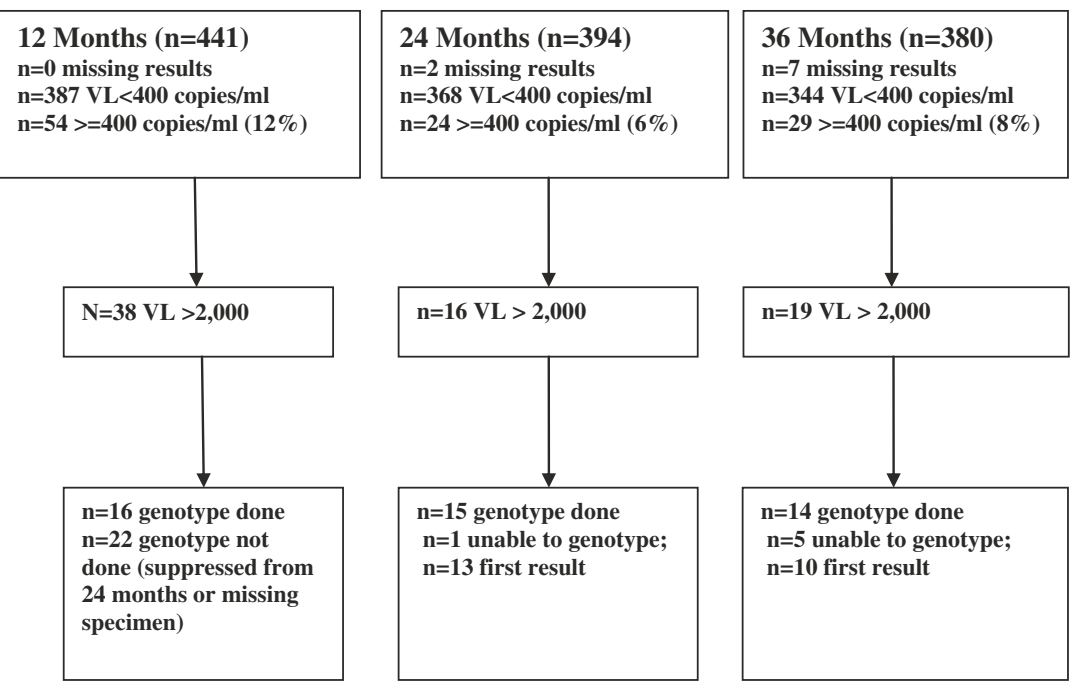

Figure 1 a. Enrollment and accrual: Immunologically monitored clients. b. Virologically monitored clients. VL: Viral Load.

duration of failure which could influence the mutation patterns observed.

Despite the limitations mentioned pertaining to study design, baseline characteristics, treatment setting and study staff were all similar. Virologic and immunologic characteristics at 36 months of follow-up were also similar between the two groups. . We believe the reduced rate of resistance, particularly to NRTIs, observed among VLM clients is explained by the frequent VL monitoring and ability to detect and allow caregivers to discuss adherence and possibly switch to second-line regimens prior to the development of extensive drug resistance. Our results support ongoing efforts to improve adherence counseling strategies as treatment programs continue to scale up access. Our study was not designed to look at longer term mortality benefits between our VLM and IM clients, however, a recent multi-country study conducted in Malawi, Zambia and South Africa suggested that the lower rate of mortality observed among South African ART programs could be explained in part by the presence of VL monitoring and timely switch to second-line regimens [20]. As ART programs continue to scale up in RLS and funding constraints limit the choices of monitoring options, the debate over how best to monitor clients will continue. Some controlled clinical trials have failed to show a benefit of VL 
Table 2 Resistance over time for virologically monitored (VLM) clients

\begin{tabular}{|c|c|c|c|}
\hline & $\begin{array}{c}\text { Month } 12 \\
(\mathrm{~N}=16)\end{array}$ & $\begin{array}{c}\text { Month } 24 \\
(\mathrm{~N}=13)\end{array}$ & $\begin{array}{c}\text { Month } 36 \\
(\mathrm{~N}=10)\end{array}$ \\
\hline \multicolumn{4}{|l|}{ NRTI mutations } \\
\hline Any TAMs & $2(12)$ & $0(0)$ & $0(0)$ \\
\hline 1 TAMs & $1(6)$ & $0(0)$ & $0(0)$ \\
\hline 2 TAMs & $1(6)$ & $0(0)$ & $0(0)$ \\
\hline 3+ TAMs & $0(0)$ & $0(0)$ & $0(0)$ \\
\hline $41 \mathrm{~L}$ & $0(0)$ & $0(0)$ & $0(0)$ \\
\hline $65 R$ & $1(6)$ & $1(8)$ & $1(10)$ \\
\hline $67 N$ & $1(6)$ & $0(0)$ & $0(0)$ \\
\hline 70R & $1(6)$ & $0(0)$ & $0(0)$ \\
\hline $184 \mathrm{I} / \mathrm{V}$ & $7(44)$ & $8(62)$ & $4(40)$ \\
\hline $210 W$ & $0(0)$ & $0(0)$ & $0(0)$ \\
\hline $215 F / Y$ & $1(6)$ & $0(0)$ & $0(0)$ \\
\hline \multicolumn{4}{|l|}{ NNRTI mutations } \\
\hline Any NNRTI mutation & $8(50)$ & $9(69)$ & $6(60)$ \\
\hline 901 & $0(0)$ & $0(0)$ & $0(0)$ \\
\hline $98 \mathrm{G}$ & $0(0)$ & $0(0)$ & $0(0)$ \\
\hline 101E & $1(6)$ & $0(0)$ & $0(0)$ \\
\hline $103 N$ & $3(19)$ & $3(23)$ & $3(30)$ \\
\hline 1081 & $0(0)$ & $0(0)$ & $2(20)$ \\
\hline $138 \mathrm{~A} / \mathrm{G}$ & $0(0)$ & $0(0)$ & $0(0)$ \\
\hline $179 D$ & $0(0)$ & $0(0)$ & $0(0)$ \\
\hline $181 \mathrm{C} / \mathrm{I}$ & $0(0)$ & $4(31)$ & $2(20)$ \\
\hline $188 \mathrm{~L} / \mathrm{H}$ & $0(0)$ & $1(8)$ & $0(0)$ \\
\hline $190 \mathrm{~A} / \mathrm{S}$ & $3(19)$ & $2(15)$ & $1(10)$ \\
\hline $225 \mathrm{H}$ & $0(0)$ & $0(0)$ & $0(0)$ \\
\hline $230 \mathrm{~L}$ & $0(0)$ & $0(0)$ & $0(0)$ \\
\hline
\end{tabular}

Bold: major mutations.

VLM: Virologically monitored; NRTI: nucleoside reverse transcriptase inhibitor; TAMs: thymidine analogue mutations; NNRTI: non-nucleoside reverse transcriptase inhibitor.

monitoring over immunologic or clinical monitoring $[21,22]$. Retention and adherence in these clinical trials is clearly higher than in the operational context of this study and may not be generalizable to operational contexts. Cost benefit studies have also suggested that allocating resources to earlier initiation of ART could provide more benefit than routine VL monitoring [23]. We feel it is equally important to evaluate monitoring strategies in a real world setting outside the support of a clinical trial where participants receive maximum adherence support and therefore risks of virologic failure are minimized. It is also important not to consider only an all or none approach to VL monitoring and consider new strategies using periodic VL monitoring at fixed time points during follow-up as a recent study in Thailand found cost-effective among pediatric clients [24].
Table 3 Resistance comparing IM versus VLM patients

\begin{tabular}{|c|c|c|}
\hline & $\begin{array}{c}\text { IM } \\
(N=70)\end{array}$ & $\begin{array}{c}\text { VLM } \\
(\mathrm{N}=39)\end{array}$ \\
\hline \multicolumn{3}{|l|}{ NRTI mutations } \\
\hline Any TAMs & $34(49)$ & $2(5)$ \\
\hline 1 TAMs & $13(19)$ & $1(3)$ \\
\hline 2 TAMs & $14(20)$ & $1(3)$ \\
\hline 3+ TAMs & $7(10)$ & $0(0)$ \\
\hline $41 \mathrm{~L}$ & $8(11)$ & $0(0)$ \\
\hline $65 R$ & $1(1)$ & $3(8)$ \\
\hline $67 \mathrm{~N}$ & $11(16)$ & $1(3)$ \\
\hline 70R & $13(19)$ & $1(3)$ \\
\hline $184 \mathrm{I} / \mathrm{V}$ & $61(87)$ & $19(49)$ \\
\hline $210 \mathrm{~W}$ & $1(1)$ & $0(0)$ \\
\hline $215 \mathrm{~F} / \mathrm{Y}$ & $25(36)$ & $1(3)$ \\
\hline \multicolumn{3}{|l|}{ NNRTI mutations } \\
\hline Any NNRTI mutation & $63(90)$ & $23(59)$ \\
\hline 901 & $5(7)$ & $0(0)$ \\
\hline $98 \mathrm{G}$ & $5(7)$ & $0(0)$ \\
\hline $101 \mathrm{E}$ & $8(11)$ & $1(3)$ \\
\hline $103 N$ & $23(33)$ & $10(26)$ \\
\hline 1081 & $10(14)$ & $2(5)$ \\
\hline $138 \mathrm{~A} / \mathrm{G}$ & $3(4)$ & $0(0)$ \\
\hline $179 D$ & $1(1)$ & $0(0)$ \\
\hline $181 \mathrm{C} / \mathrm{I}$ & $22(31)$ & $6(15)$ \\
\hline $188 \mathrm{~L} / \mathrm{H}$ & $3(4)$ & $1(3)$ \\
\hline $190 \mathrm{~A} / \mathrm{S}$ & $13(19)$ & $6(15)$ \\
\hline $225 \mathrm{H}$ & $6(9)$ & $0(0)$ \\
\hline $230 \mathrm{~L}$ & $1(1)$ & $0(0)$ \\
\hline
\end{tabular}

Bold: major mutations.

IM: Immunologically monitored; VLM: Virologically monitored; NRTI: nucleoside reverse transcriptase inhibitor; TAMS: thymidine analogue mutations; NNRTI: non-nucleoside reverse transcriptase inhibitor.

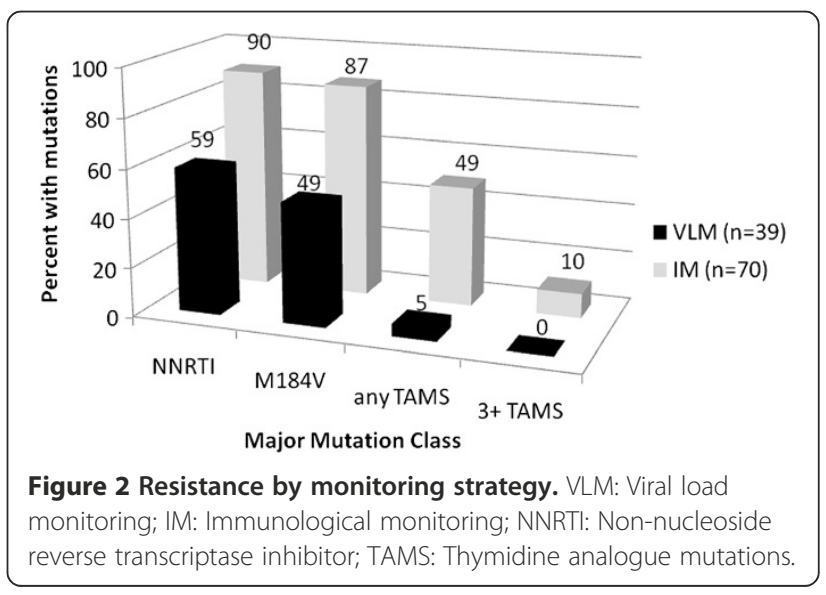




\section{Conclusions}

Access to viral load monitoring remains a priority for countries continuing to scale up ART in order to limit the emergence of drug resistance. Further studies are warranted to evaluate novel monitoring strategies that minimize resistance, maximize positive clinical outcomes, recognizing cost constraints faced by programs challenged by ever increasing numbers needing HIV treatment.

\section{Competing interests}

The authors declared that they have no competing interests.

\section{Authors' contributions}

SJR, HK, TCQ and AK contributed to study design, participant recruitment and follow-up, and final analysis. KN contributed to study conduct and statistical analysis. YCM, MK and BC contributed to participant recruitment, study conduct and analysis. In performed laboratory assays and contributed to analysis. All authors contributed to manuscript writing. All authors read and approved the final manuscript.

\section{Acknowledgements}

We wish to thank the clients and staff at the Infectious Diseases Institute and also the laboratory staff at the Joint Clinical Research Center CFAR lab who supported this research.

\section{Funding}

This research was supported by the Division of Intramural Research, National Institute of Allergy and Infectious Diseases, National Institutes of Health. This project has been funded in whole or in part with federal funds from the National Cancer Institute, National Institutes of Health, under Contract No. HHSN261200800001E. The content of this publication does not necessarily reflect the views or policies of the Department of Health and Human Services, nor does mention of trade names, commercial products, or organizations imply endorsement by the U.S. Government.

\section{Author details}

'Division of Intramural Research, National Institute of Allergy and Infectious Diseases, National Institutes of Health, Bethesda, MD, USA. ${ }^{2}$ Johns Hopkins University School of Medicine, Baltimore, MD, USA. ${ }^{3}$ Uganda Ministry of Health, Kampala, Uganda. ${ }^{4}$ Department of Microbiology, Makerere University College of Health Sciences, Kampala, Uganda. ${ }^{5}$ Clinical Research Directorate/ Clinical Monitoring Research Program, SAIC-Frederick, Inc, Frederick National Laboratory for Cancer Research, Frederick, MD 21702, USA. ${ }^{6}$ Infectious Diseases Institute, Makerere University College of Health Sciences, Kampala, Uganda. ${ }^{7}$ Joint Clinical Research Center, Kampala, Uganda. ${ }^{8} \mathrm{NIAID} / \mathrm{NIH}$ ICER Program, P.O. Box 7007, Kampala, Uganda.

Received: 24 May 2012 Accepted: 20 December 2012 Published: 27 December 2012

\section{References}

1. UNAIDS: Report on the Global AIDS Epidemic 2010. 2010. Ref Type: Report http://www.unaids.org/en/media/unaids/contentassets/documents/ unaidspublication/2010/20101123_globalreport_en\%5B1\%5D.pdf.

2. Calmy A, Ford N, Hirschel B, Reynolds SJ, Lynen L, Goemaere E, et al: HIV viral load monitoring in resource-limited regions: optional or necessary? Clin Infect Dis 2007, 44:128-134.

3. Castelnuovo B, Kiragga A, Schaefer P, Kambugu A, Manabe Y: High rate of misclassification of treatment failure based on WHO immunological criteria. AIDS 2009, 23:1295-1296.

4. Mee P, Fielding KL, Charalambous S, Churchyard GJ, Grant AD: Evaluation of the WHO criteria for antiretroviral treatment failure among adults in South Africa. AIDS 2008, 22:1971-1977.

5. Moore DM, Awor A, Downing R, Kaplan J, Montaner JS, Hancock J, et al: CD4+ T-cell count monitoring does not accurately identify
HIV-infected adults with virologic failure receiving antiretroviral therapy. J Acquir Immune Defic Syndr 2008, 49:477-484.

6. Reynolds SJ, Nakigozi G, Newell K, Ndyanabo A, Galiwongo R, Boaz I, et al: Failure of immunologic criteria to appropriately identify antiretroviral treatment failure in Uganda. AIDS 2009, 23:697-700.

7. van Oosterhout JJ, Brown L, Weigel R, Kumwenda JJ, Mzinganjira D, Saukila N, et al: Diagnosis of antiretroviral therapy failure in Malawi: poor performance of clinical and immunological WHO criteria. Trop Med Int Health 2009, 14:856-861.

8. Hosseinipour MC, van Oosterhout JJ, Weigel R, Phiri S, Kamwendo D, Parkin N, et al: The public health approach to identify antiretroviral therapy failure: high-level nucleoside reverse transcriptase inhibitor resistance among Malawians failing first-line antiretroviral therapy. AIDS 2009, 23:1127-1134.

9. Sigaloff $\mathrm{KC}$, Hamers $\mathrm{RL}$, Wallis $\mathrm{CL}$, Kityo $\mathrm{C}$, Siwale $\mathrm{M}$, Ive $\mathrm{P}$, et al: Unnecessary Antiretroviral Treatment Switches and Accumulation of HIV Resistance Mutations; Two Arguments for Viral Load Monitoring in Africa. J Acquir Immune Defic Syndr 2011, 58:23-31.

10. Boucher CA, O'Sullivan E, Mulder JW, Ramautarsing C, Kellam P, Darby $G$, et al: Ordered appearance of zidovudine resistance mutations during treatment of 18 human immunodeficiency viruspositive subjects. J Infect Dis 1992, 165:105-110.

11. Lyagoba F, Dunn DT, Pillay D, Kityo C, Robertson V, Tugume S, et al: Evolution of drug resistance during 48 weeks of zidovudine/ lamivudine/tenofovir in the absence of real-time viral load monitoring. J Acquir Immune Defic Syndr 2010, 55:277-283.

12. Sigaloff KC, Ramatsebe T, Viana R, Wit TF, Wallis CL, Stevens WS: Accumulation of HIV Drug Resistance Mutations in Patients Failing First-Line Antiretroviral Treatment in South Africa. AIDS Res Hum Retroviruses 2011, 28(2):171-175.

13. World Health Organization: Antiretroviral Therapy for HIV Infection in Adults and Adolescents in Resource-Limited Settings: Towards Universal Access. Recommendations for a public health approach 2006; 2006. Ref Type: Online Source.

14. Kiragga AN, Castelnuovo B, Nakanjako D, Manabe YC: Baseline severe anaemia should not preclude use of zidovudine in antiretroviraleligible patients in resource-limited settings. J Int AIDS Soc 2010, 13:42.

15. Castelnuovo B, Manabe $Y C$, Kiragga A, Kamya M, Easterbrook P, Kambugu A: Cause-specific mortality and the contribution of immune reconstitution inflammatory syndrome in the first 3 years after antiretroviral therapy initiation in an urban African cohort. Clin Infect Dis 2009, 49:965-972.

16. Coetzee D, Boulle A, Hildebrand K, Asselman V, Van CG, Goemaere E: Promoting adherence to antiretroviral therapy: the experience from a primary care setting in Khayelitsha, South Africa. AIDS 2004, 18(Suppl 3):S27-S31.

17. Johnson VA, Calvez V, Gunthard HF, Paredes R, Pillay D, Shafer R, et al: 2011 update of the drug resistance mutations in HIV-1. Top Antivir Med 2011, 19:156-164.

18. Vidya M, Saravanan S, Uma S, Kumarasamy N, Sunil SS, Kantor R, et al: Genotypic HIV type-1 drug resistance among patients with immunological failure to first-line antiretroviral therapy in south India. Antivir Ther 2009, 14:1005-1009.

19. Hosseinipour MC, Kumwenda JJ, Weigel R, Brown LB, Mzinganjira D, Mhango B, et al: Second-line treatment in the Malawi antiretroviral programme: high early mortality, but good outcomes in survivors, despite extensive drug resistance at baseline. HIV Med 2010, 11:510-518

20. Keiser O, Chi BH, Gsponer T, Boulle A, Orrell C, Phiri S, et al: Outcomes of antiretroviral treatment in programmes with and without routine viral load monitoring in southern Africa. AIDS 2011, 25:1761-1769.

21. Laurent C, Kouanfack C, Laborde-Balen G, Aghokeng AF, Mbougua JB, Boyer S, et al: Monitoring of HIV viral loads, CD4 cell counts, and clinical assessments versus clinical monitoring alone for antiretroviral therapy in rural district hospitals in Cameroon (Stratall ANRS 12110/ ESTHER): a randomised non-inferiority trial. Lancet Infect Dis 2011, 11:825-833.

22. Mugyenyi P, Walker AS, Hakim J, Munderi P, Gibb DM, Kityo C, et al: Routine versus clinically driven laboratory monitoring of HIV 
antiretroviral therapy in Africa (DART): a randomised non-inferiority trial. Lancet 2010, 375:123-131.

23. Braithwaite RS, Nucifora KA, Yiannoutsos CT, Musick B, Kimaiyo $S$,

Diero $L$, et al: Alternative antiretroviral monitoring strategies for HIV-infected patients in east Africa: opportunities to save more lives? J Int AIDS Soc 2011, 14:38.

24. Schneider K, Puthanakit T, Kerr S, Law MG, Cooper DA, Donovan B, et al: Economic evaluation of monitoring virologic responses to antiretroviral therapy in HIV-infected children in resource-limited settings. AIDS 2011, 25:1143-1151.

\section{doi:10.1186/1471-2334-12-381}

Cite this article as: Reynolds et al:: Virologic versus immunologic monitoring and the rate of accumulated genotypic resistance to firstline antiretroviral drugs in Uganda. BMC Infectious Diseases 2012 12:381.

\section{Submit your next manuscript to BioMed Central and take full advantage of:}

- Convenient online submission

- Thorough peer review

- No space constraints or color figure charges

- Immediate publication on acceptance

- Inclusion in PubMed, CAS, Scopus and Google Scholar

- Research which is freely available for redistribution 